\title{
Left atrial endocardial dysfunction and platelet activation in patients with atrial fibrillation and mitral stenosis
}

\author{
Zhi-Qiang Luo, MD, ${ }^{a}$ Xing-Hai Hao, MD, ${ }^{b}$ Jin-Hua Li, MD, ${ }^{c}$ Jiang Dai, MD, ${ }^{c}$ Ke-Ye Liu, MD, ${ }^{a}$ and \\ Yong-Qiang Lai, MD ${ }^{\mathrm{c}}$
}

Objective: This study demonstrated left atrial endocardial dysfunction and platelet activation in patients with atrial fibrillation and mitral stenosis.

\begin{abstract}
Methods: Study included 80 patients with mitral stenosis and atrial fibrillation (40 each with and without left atrial thrombosis), 15 healthy volunteers, and 10 left atrial appendage (LAA) specimens from donor hearts. Blood samples were collected through peripheral vein and left atrium, with peripheral blood samples of volunteers as controls. LAA specimens were collected during operations. LAA expressions of von Willebrand factor (vWF) and P-selectin were determined immunohistochemically; plasma concentrations were measured by enzyme-linked immunosorbent assay. LAA expressions of vWF and P-selectin genes in were quantitated with real-time fluorescent quantitative polymerase chain reaction.
\end{abstract}

Results: The difference in vWF and P-selectin plasma levels between left atrial and peripheral venous blood was not significant; however, peripheral plasma levels of vWF and P-selectin were significantly higher in those with thrombosis than without thrombosis, which in turn were higher than in healthy subjects. Both vWF and P-selectin proteins were stained in both left atrial endocardium and cardiomyocytes. The normalized $\mathrm{vWF}$ gene expression relative to control was 3.04 in patients with thrombosis and 2.16 in those without thrombosis $(P<.01)$. The difference in P-selectin gene expressions among the groups was not significant.

Conclusions: No differences were observed in plasma levels of vWF and P-selectin between left atrial and peripheral venous blood. Over expression of vWF gene in LAA may contribute to increased plasma vWF levels. P-selectin and vWF together may play a role in thrombosis. (J Thorac Cardiovasc Surg 2014;148:1970-6)

See related commentary on pages 1976-7.

Rheumatic mitral stenosis (MS) combined with atrial fibrillation (AF) is often associated with left atrial thrombosis (LAT). The incidence of LAT in patients with MS confirmed by surgical operation ranges from 17.41 to $24.33 \% .^{1,2}$ Early studies have demonstrated that left atrial endocardial injury and dysfunction and abnormal platelet

From the Department of Cardiac Surgery, ${ }^{\mathrm{a}}$ Affiliated Hospital of HeBei University, Baoding, Hebei, China; the Department of Cardiac Surgery, ${ }^{\mathrm{b}}$ The Third Hospital, Beijing University, Beijing, China; and the Division of Cardiac Surgery, ${ }^{c}$ Beijing Anzhen Hospital, Capital Medical University, Beijing, China.

Supported by Beijing Municipal National Science Foundation (7102049) and Capital Chinese Medicine Technology Department Foundation (SF-2011-2-17).

Disclosures: Authors have nothing to disclose with regard to commercial support.

Z.-Q.L., X.-H.H., and J.-H.L. contributed equally to this work.

Received for publication Nov 12, 2012; revisions received May 25, 2013; accepted for publication June 6, 2013; available ahead of print Jan 13, 2014.

Address for reprints: Yong-Qiang Lai, MD, Department of Cardiac Surgery, Beijing Anzhen Hospital, Capital Medical University, 36 Wuluju Chaoyang district,

Beijing, China, 100029 (E-mail: yongqianglai@yahoo.com).

$0022-5223 / \$ 36.00$

Copyright (c) 2014 by The American Association for Thoracic Surgery

http://dx.doi.org/10.1016/j.jtcvs.2013.06.064 activation are present in patients with $\mathrm{LAT}^{3-5}$; however, the mechanisms of platelet activation and endocardial dysfunction and their relationships with LAT are still unclear. Plasma von Willebrand factor (vWF) and P-selectin are considered as biomarkers of endothelium injury and dysfunction and platelet activation. ${ }^{6}$ They are involved in platelet adhesion and aggregation and thus start the hemostatic process. To evaluate the roles of left atrial endocardial dysfunction and platelet activation in LAT, the changes of plasma levels of vWF, P-selectin, and their gene expressions were studied in left atrial appendage (LAA) samples from patients with MS and AF.

\section{MATERIALS AND METHODS}

The study was performed with the permission of our institutional review board. Individual consents for surgery and collection of LAA and blood samples were obtained before operation.

\section{Patients and Sampling}

Eighty patients with MS combined with long-standing, persistent AF (40 patients with LAT, 40 without) and 15 healthy subjects were studied. Patients with chronic obstructive pulmonary disease, hematologic diseases, stroke, or hepatic or renal dysfunction were excluded. LAT was diagnosed during surgery. Transthoracic echocardiography was performed for all the patients before surgery. All 80 patients underwent mitral valve replacement and bipolar radiofrequency ablation modification of the maze III operation, 


\section{Abbreviations and Acronyms \\ $\mathrm{AF} \quad=$ atrial fibrillation \\ $\mathrm{Ct}=$ threshold cycle \\ LAT $=$ left atrial thrombosis \\ MS $=$ mitral stenosis \\ RT-PCR $=$ real-time polymerase chain reaction}

as described by Doty and colleagues. ${ }^{7}$ The LAA was excised during the operation. Blood samples were collected through a peripheral vein and the left atrium during the operation. In healthy subjects, only peripheral blood samples were collected as controls. LAA specimens were obtained during the radiofrequency ablation in the patients with MS and AF. Ten LAA specimens were obtained from donor hearts for heart transplant as controls.

\section{Measurements of Plasma Concentrations of vWF and P-selectin}

Plasma concentrations of $\mathrm{vWF}$ and P-selectin were measured by enzyme-linked immunosorbent assay. Blood samples were stored in EDTA acid anticoagulation tubes. Plasma was extracted by centrifuging for 15 minutes at $3000 \mathrm{rpm}$. Samples were stored at $-80^{\circ} \mathrm{C}$ for study. Human vWF ELISA kit (R\&D Systems Inc, Minneapolis, Minn) and human P-selectin ELISA kit (R\&D Systems Inc) were used for detection and measurement of vWF and P-selectin, respectively.

\section{Detection of vWF and P-selectin Proteins in LAA Tissue}

Detection of vWF and P-selectin protein in LAA tissue was performed immunohistochemically. LAA specimens were immediately fixed in $4 \%$ paraformaldehyde for 24 hours at room temperature. The LAA tissue was embedded in paraffin. Serial cryostat sections ( $4 \mu \mathrm{m}$ in thickness) were cut from each tissue block and were immunostained with the avidin-biotin immunoperoxidase method. Briefly, sections were incubated with the following solutions in the following order: (1) $2 \%$ hydrogen peroxide for 30 minutes to block endogenous peroxidase activity; (2) $0.3 \%$ Triton X-100 (The Dow Chemical Company, Midland, Mich) for 15 minutes to permeabilize the membrane; (3) $10 \%$ normal goat serum for 15 minutes at room temperature to reduce nonspecific antiserum binding; (4) primary antisera for 16 hours at $4^{\circ} \mathrm{C}$ (1:100 for vWF and 1:500 for P-selectin); (5) biotinylated goat antimouse at $37^{\circ} \mathrm{C}$ for 45 minutes; and (6) avidin-biotinylated horseradish peroxidase complex (Abcam Inc, Cambridge, Mass) at $37^{\circ} \mathrm{C}$ for 15 minutes. Immunoreactive sites were visualized by incubation with $0.025 \%$ 3,3-diaminobenzidine and $0.01 \%$ hydrogen peroxide for 3 minutes. Phosphate-buffered saline solution ( $\mathrm{pH}$ 7.4) was used to dilute each solution and to wash the sections 3 times between each step. Tissue sections were counterstained with hematoxylin. All tissue sections were analyzed under an optical microscope.

\section{Study of vWF and P-selectin Gene Expressions in LAA Tissue}

Expressions of vWF and P-selectin genes in LAA tissue were studied with real-time polymerase chain reaction (RT-PCR). Once collected, LAA specimens were immediately frozen in liquid nitrogen. Total RNA was extracted from each tissue sample with Trizol reagent (Invitrogen Inc, Life Technologies Corporation, Grand Island, NY) according to the manufacturer's instructions. Aliquots of the total RNA samples were diluted in TE buffer, and the concentration of each sample was measured at $260 \mathrm{~nm}\left(\mathrm{~A}_{260}\right)$ with a spectrophotometer (BioPhotometer, Eppendorf AG, Hamburg, Germany). Gene expression analysis was performed only when the $\mathrm{A}_{260} / \mathrm{A}_{280}$ ratios ranged between 1.9 and 2.1, and the integrity of each RNA sample was confirmed by agarose gel electrophoresis.

RNA was transcribed to complementary DNA with oligonucleotide primers and subsequently amplified with an M-MLV reverse transcription kit (Takara Bio Inc, Ötsu Japan) according to the manufacturer's instructions.

First-strand complementary DNA samples were subjected to quantitative PCR amplification to measure the concentrations of RNA transcripts. RT-PCR was performed with the ABI PRISM 7500 Sequence Detection System (Applied Biosystems, Life Technologies) and SYBR Green I (Life Technologies) chemistry. Each of the complementary DNA samples was examined to assess gene expression levels for the vWF gene, the P-selectin gene, and the glyceraldehyde 3phosphate dehydrogenase gene (housekeeping gene). Oligonucleotide primers for quantification of vWF, P-selectin, and glyceraldehyde 3phosphate dehydrogenase gene transcripts were designed with Primer Express software (version 1.5; PerkinElmer Inc, Waltham, Mass). All the PCR analyses were performed with the following cycling conditions: 15 minutes at $95^{\circ} \mathrm{C}$ (initial melt), followed by 40 cycles of 15 seconds at $95^{\circ} \mathrm{C}, 1$ minute at $60^{\circ} \mathrm{C}$ and 15 seconds at $72^{\circ} \mathrm{C}$. The specificity of the primers was verified by $2 \%$ agarose gel electrophoresis of the amplicons.

The $2^{-\Delta \Delta \mathrm{Ct}}$ method was used to investigate the relative changes of vWF and P-selectin gene expressions in LAA. The quantification of gene expression was determined by measuring the threshold cycle $(\mathrm{Ct})$ for each gene and calculated as follows: Target gene expression $=2^{-\Delta \Delta \mathrm{Ct}}$, where $\Delta \Delta \mathrm{Ct}=\Delta \mathrm{Ct}_{\text {Study group }}-\Delta \mathrm{Ct}_{\text {Control group }}$, with $\Delta \mathrm{Ct}$ derived as $\mathrm{Ct}_{\text {Target gene }}-\mathrm{Ct}_{\text {Housekeeping gene }}$.

\section{Statistical Analysis}

Quantitative data are presented as mean $\pm \mathrm{SD}$. The difference between groups was determined by $t$ test and 1-way analysis of variance with the SPSS statistical software package (version 17.0; IBM Corporation, Armonk, NY).

\section{RESULTS}

The clinical characteristics are summarized in Table 1. Relative to healthy subjects, patients with MS had bigger left atrium. Among patients with MS, those with LAT had a bigger left atrium, a smaller mitral valve orifice, and a longer duration of AF than did those without LAT.

Study on the sections under optical microscopy showed that the LAA endocardium of MS patient with AF was rough and was clearly thickened by randomly arranged new cells. Chronic fiber scarring was present in the endocardium of patients with LAT, accompanied by new endothelial cell migration and inflammatory cell infiltration (Figures 1 and 2). Immunostained $\mathrm{vWF}$ and P-selectin proteins were observed in LAA samples from both donor hearts (Figures 3 and 4) and patients with MS and AF (Figures 5 and 6) in both the endocardium and the cardiomyocytes. The $\mathrm{vWF}$ protein was present near the nuclei, whereas the P-selectin protein was found near the edge of cardiomyocytes. The stainings of vWF and $\mathrm{P}$-selectin proteins were weak in donor hearts.

The difference in plasma levels of vWF and P-selectin between left atrium and peripheral venous blood was not 
TABLE 1. Clinical data

\begin{tabular}{lccc}
\hline & $\begin{array}{c}\text { With } \\
\text { LAT } \\
(\mathbf{n}=\mathbf{4 0})\end{array}$ & $\begin{array}{c}\text { Without } \\
\text { LAT } \\
(\mathbf{n}=\mathbf{4 0})\end{array}$ & $\begin{array}{c}\text { Control } \\
(\mathbf{n}=\mathbf{1 5})\end{array}$ \\
\hline Age (y) & $53.75 \pm 8.38$ & $51.95 \pm 7.46$ & $50.93 \pm 10.9$ \\
Male & $19(47.50 \%)$ & $18(45.00 \%)$ & $8(53.3 \%)$ \\
Body & $60.21 \pm 8.52$ & $65.52 \pm 10.10$ & $61.95 \pm 9.52$ \\
$\quad$ weight (kg) & & & \\
Hypertension & $7(17.5 \%)$ & $6(15 \%)$ & 0 \\
Diabetes & $4(10 \%)$ & $5(12.5 \%)$ & - \\
Duration of & $69.80 \pm 29.54 *$ & $54.83 \pm 23.30$ & \\
$\quad$ AF (mo) & & & $1.00 \pm 0.00$ \\
NYHA & $2.68 \pm 0.38$ & $2.35 \pm 0.34$ & \\
$\quad$ classification & & & \\
Platelets & $185.70 \pm 51.19$ & $195.10 \pm 41.39$ & $180.6 \pm 22.36$ \\
$\quad(109$ cells/L) & & & \\
Transthoracic echocardiography & & \\
$\quad$ LVEF (\%) & $58.30 \% \pm 7.61 \%$ & $60.37 \% \pm 6.54 \%$ & $61.46 \% \pm 3.88 \%$ \\
LVEDD (mm) & $43.92 \pm 4.51$ & $45.02 \pm 4.35$ & $45.93 \pm 4.04$ \\
LAD (mm) & $51.05 \pm 7.54 \dagger$ & $45.45 \pm 6.32 \ddagger$ & $33.62 \pm 2.47$ \\
MVA (cm $\left.{ }^{2}\right)$ & $0.88 \pm 0.22 \S$ & $1.11 \pm 0.22$ & - \\
\hline
\end{tabular}

Data are mean \pm SD or number of patients. LAT, Left atrial thrombosis; $A F$, atrial fibrillation; NYHA, New York Heart Association; $L V E F$, left ventricular ejection fraction; $L V E D D$, left ventricular end-diastolic diameter; $L A D$, left atrial dimension; $M V A$, mitral valve orifice area. $* P=.008$ versus patients without thrombosis. $\dagger P<.001$ versus patients without thrombosis and versus control. $\ddagger P<.001$ versus patients with thrombosis and versus control. $\S P<.001$ versus patients without thrombosis and versus control.

significant in patients with MS with or without LAT. The peripheral plasma levels of $\mathrm{vWF}$ and P-selectin in patients with MS and LAT were significantly higher than those in patients with MS without LAT and those in healthy subjects. The plasma levels of vWF and P-selectin concentration in patients with MS without LAT were also higher than those in healthy subjects (Table 2).

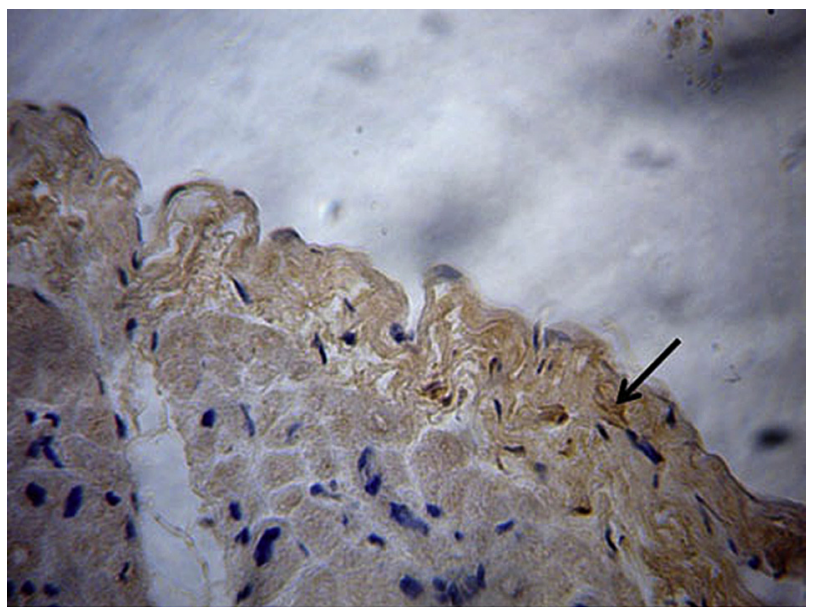

FIGURE 1. Immunohistochemical staining for vWF in an LAA sample from a patient with MS and AF. The endocardium is rough and thickened, and immunostained $\mathrm{vWF}$ protein can be observed (arrow). Original magnification $\times 200$.

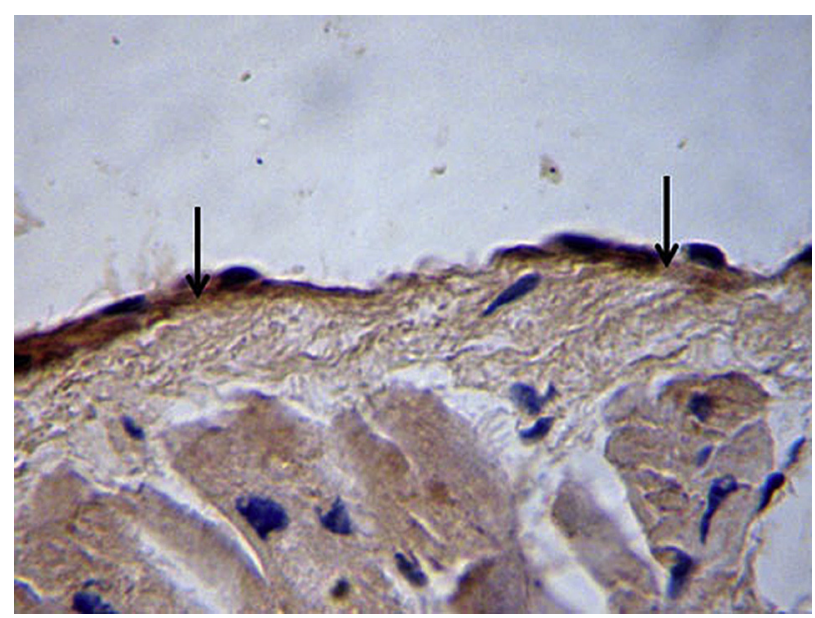

FIGURE 2. Immunohistochemical staining for P-selectin in an LAA sample from a patient with MS and AF. The endocardium is rough and thickened, and immunostained P-selectin protein can be observed (arrows). Original magnification $\times 400$.

The normalized amount of vWF gene expression relative to control was 3.04 in patients with MS with LAT and 2.16 in those without LAT. The difference between groups was statistically significant (Table 3 ). The difference of P-selectin gene expression in LAA between different groups was not significant (Table 4).

\section{DISCUSSION}

MS combined with AF is often associated with LAT, which may induce brain and peripheral artery embolism. Cardiac enlargement, myocardial remodeling, and hemodynamic changes caused by MS and AF are important mechanism for LAT ${ }^{8,9}$ In most cases, LAT occurs first in the LAA, where blood flow is likely to form a vortex because of the LAA's structure. ${ }^{10,11}$

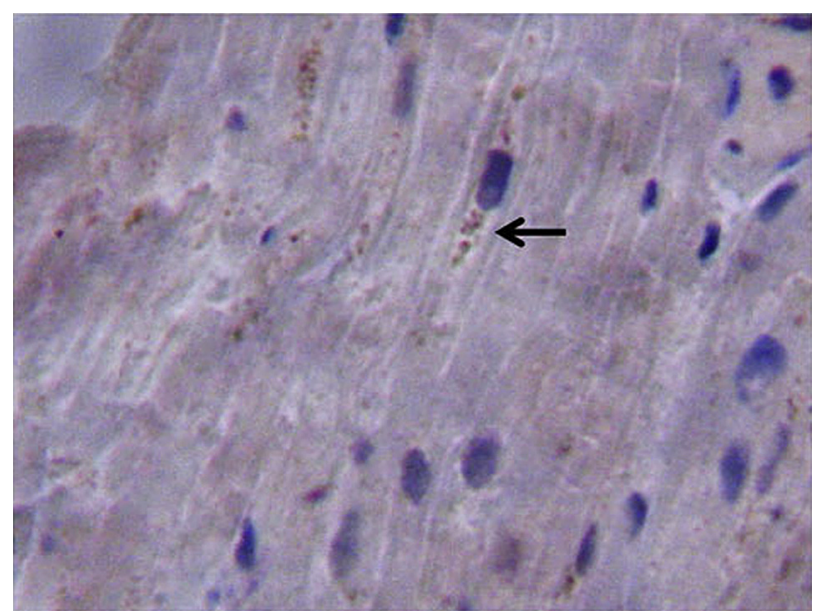

FIGURE 3. Immunohistochemical staining for vWF in an LAA sample from a donor heart. Immunostained vWF protein can be observed near the nuclei (arrow). Original magnification $\times 400$. 


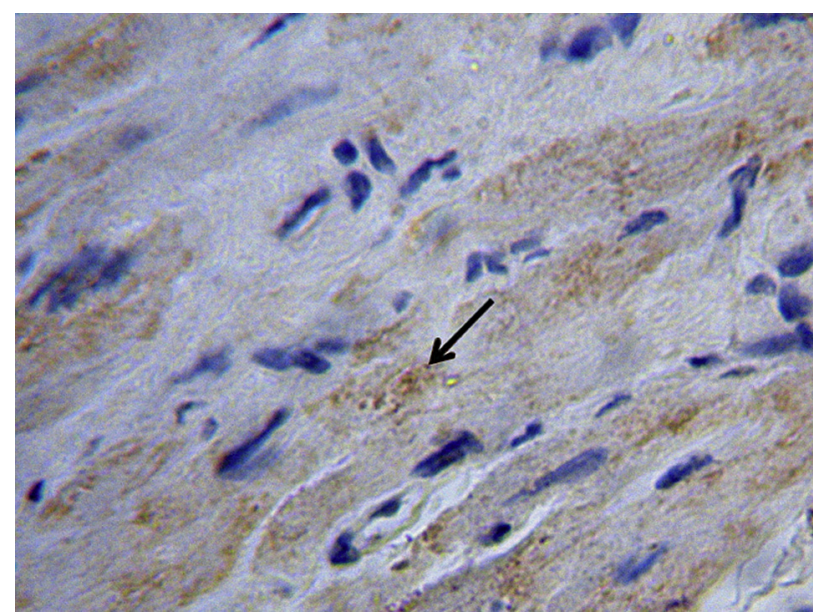

FIGURE 4. Immunohistochemical staining for P-selectin in an LAA sample from a donor heart. Immunostained P-selectin protein can be observed near the edge of cardiomyocytes (arrow). Original magnification $\times 400$.

Studies had found thickening of left atrial endocardial tissue, loss of endothelium, and thrombotic changes of the endothelium in patients with MS and AF. ${ }^{3,4}$ Abnormal platelet activation and endocardial dysfunction were observed. ${ }^{5}$ Nonetheless, the mechanism of platelet activation and endocardial dysfunction and their relationship with LAT was still unclear.

In our study, a longer duration of AF, smaller mitral valve orifice area, and larger left atrial dimension were observed in patients with LAT, which indicated the more advanced disease. As MS worsens, a progressively high left atrial pressure develops as a result of the diastolic gradient between the left atrium and ventricle. The high left atrial pressure gradually leads to left atrial enlargement and AF.

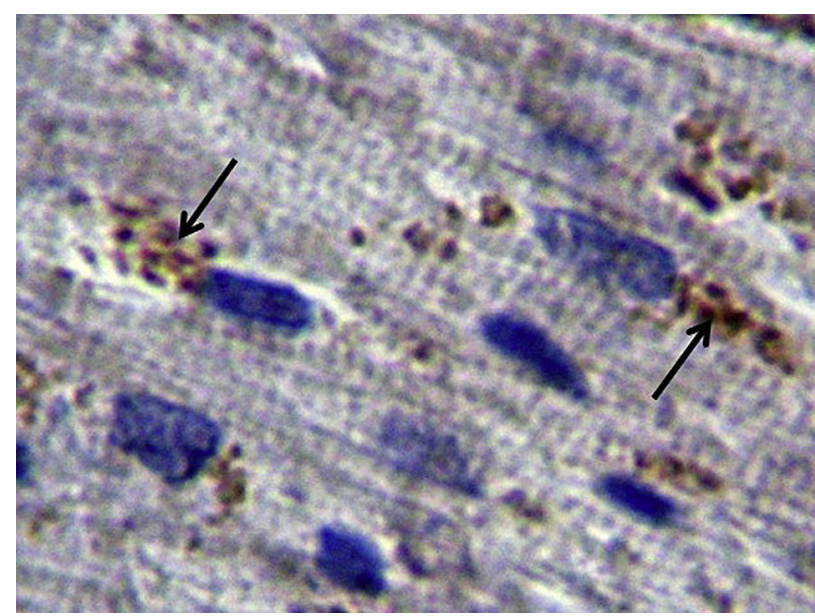

FIGURE 5. Immunohistochemical staining for vWF in an LAA sample from patient with MS and AF. Immunostained $\mathrm{vWF}$ protein can be observed near the nuclei (arrows). Original magnification $\times 400$.

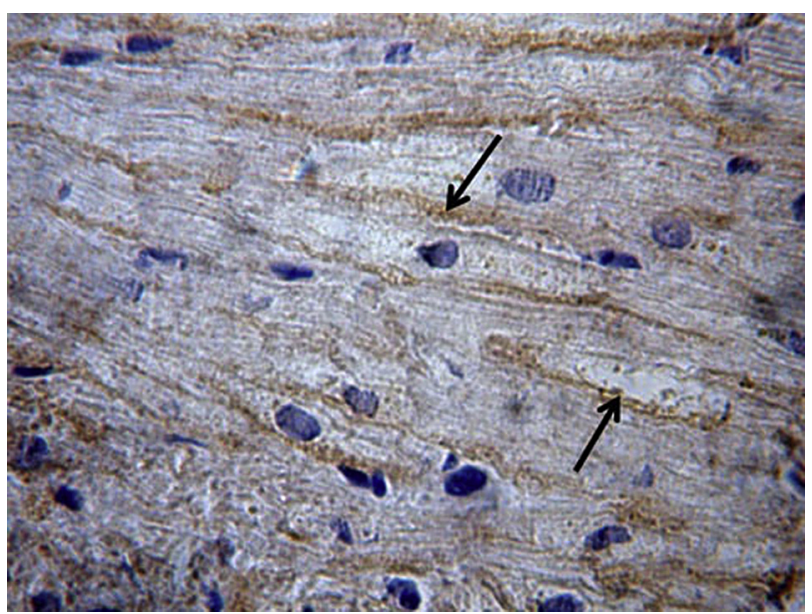

FIGURE 6. Immunohistochemical staining for P-selectin in an LAA sample from a patient with and AF. Immunostained P-selectin protein can be observed near the edge of cardiomyocytes (arrows). Original magnification $\times 400$.

The degree of left atrial enlargement correlates with the duration of stenotic lesion and involvement by the underlying rheumatic inflammatory process. ${ }^{12} \mathrm{AF}$ leads to loss of left atrial contraction, thus increasing the blood stasis in left atrium secondary to MS. Furthermore, AF leads to atrial remodeling and endocardial damage, which increases the risk of thrombosis in the LAA. ${ }^{13}$

The endothelial system is the largest autocrine, endocrine, and paracrine organ in human body. Endothelial cells secrete various vasoactive substances, which may play an important role in the regulation of vascular tone, inflammation, lipid metabolism, and particularly in thrombosis. ${ }^{14,15}$ Goldsmith and colleagues ${ }^{3}$ found endocardial damage and potentially thrombogenic changes in the atrial appendages of patients with mitral valve disease. They thought these anatomic appearances could contribute to the risk of intra-atrial thrombus formation in patients with mitral valve disease, especially in the presence of $\mathrm{AF}^{3}$ Similarly, we found that the endocardium of LAA specimens from patient with MS and AF was not smooth and was obviously thickened with randomly arranged new cells. Chronic fiber scarring was present in endocardium of patients with LAT, accompanying with new endothelial cell migration and inflammatory cell infiltration. It has been shown that fibrosis of the atrial endocardium exists in patients with solitary AF; however, more atrial endocardial fibrosis is present in patients with $\mathrm{AF}$ and concomitant mitral valve disease. ${ }^{16}$

Plasma vWF is a biomarker of endothelial injury and dysfunction, mediating platelet adhesion to subendothelial collagen fibers when endothelium injury or dysfunction occurs and thus initiating the hemostasis pathway. ${ }^{17,18}$ Left atrial endothelial dysfunction occurs in patients with MS and AF, so vWF may play an important role in the formation of LAT. 
TABLE 2. Differences between groups in plasma levels of von Willebrand factor and P-selectin

\begin{tabular}{lcccccc}
\hline & \multicolumn{2}{c}{ Patients with LAT $(\mathbf{n}=\mathbf{4 0})$} & & \multicolumn{2}{c}{ Patients without LAT $(\mathbf{n}=\mathbf{4 0})$} & Controls $(\mathbf{n}=\mathbf{1 5})$ \\
\cline { 2 - 3 } & Left atrium & Peripheral vein & & Left atrium & Peripheral vein & Peripheral vein \\
\hline $\mathrm{vWF}(\%)$ & $121.02 \% \pm 16.77 \% *$ & $124.15 \% \pm 13.50 \% \dagger$ & & $104.57 \% \pm 12.14 \%$ & $105.42 \% \pm 7.61 \%$ & $87.25 \% \pm 11.52 \%$ \\
$\mathrm{P}-\mathrm{selectin}(\mathrm{ng} / \mathrm{mL})$ & $140.79 \pm 18.20 *$ & $137.22 \pm 16.07 \dagger$ & & $115.68 \pm 14.56$ & $111.27 \pm 16.33$ & $85.67 \pm 9.66$ \\
\hline $\begin{array}{l}\text { Data are mean } \pm \text { SD. } L A T, \text { Left atrial thrombosis; } v W F, \text { von Willebrand factor. } * P<.001 \text { versus patients without thrombosis and versus controls. } \dagger P<.001 \text { versus patients without } \\
\text { thrombosis and versus controls. }\end{array}$
\end{tabular}

P-selectin is stored in a preformed state in the Weibel-Palade bodies of endothelial cells and in the granules of platelets. In response to a variety of inflammatory or thrombogenic agents, the preformed P-selectin is mobilized to the cell surface within minutes. ${ }^{15}$ P-selectin is considered a plasma marker of platelet activation. Monitoring of plasma and platelet membrane P-selectin levels can help to elucidate the extent of platelet activation. ${ }^{19,20}$ It is involved in the adhesion of myeloid cells to activated endothelium and in the adhesion of platelets to monocytes and neutrophils. ${ }^{21}$

No significant differences in plasma levels of vWF and P-selectin concentration between the left atrium and peripheral venous blood were observed in our study. Chen and associates ${ }^{22,23}$ presented similar results in their study on patients with symptomatic MS undergoing percutaneous transluminal mitral valvuloplasty. This suggests that measurements of plasma levels of vWF and $\mathrm{P}$-selectin concentration in peripheral venous blood might reflect their plasma levels in the left atrium. The extent of endothelial injury and dysfunction and platelet activation could be evaluated by examination of blood samples from a peripheral vein; however, there is no widely accepted threshold for vWF or P-selectin levels associated with the formation of LAT.

Our results demonstrate that left atrial endothelial dysfunction and abnormal platelet activation exist in patients with MS combined with AF regardless of whether LAT is present. The increases in plasma vWF and P-selectin concentrations was more obvious in patients with LAT than in patients without LAT. It might be related to the larger left atrial dimension, smaller mitral valve orifice area, and longer duration of AF in patients with LAT. Other studies have presented similar results about changes of plasma vWF and P-selectin concentration in patients with MS. ${ }^{8,24,25}$ Reports on expressions of vWF and P-selectin in atrial endocardium are few. Kumagai and colleagues ${ }^{24}$ investigated the expressions of vWF messenger RNA and protein in the endocardium by means of immunohistochemistry and in situ hybridization. They found vWF to be overexpressed in the endocardium of the LAA. This overexpression was associated with enlarged left atrial dimensions in mitral valvular disease or increased myocyte diameters in the underlying myocardium. Endocardial overexpression of vWF may occur during the process of atrial structural remodeling, contributing to the thrombotic predilection of $\mathrm{AF}$ in association with underlying heart disease. In our study, both vWF protein and P-selectin protein were immunohistochemically stained in the endocardium of the LAA. Furthermore, vWF protein and P-selectin protein staining were also observed within cardiomyocytes, which has not been reported previously. Literature review did not find any quantitative studies on expressions of $\mathrm{vWF}$ gene and P-selectin gene in the LAA. The staining of vWF and P-selectin proteins in donor hearts seem thinner than seen in patients with MS and AF. We therefore carried out RT-PCR to examine the gene expressions of vWF and P-selectin.

To the best of our knowledge, the quantitation of gene expressions of $\mathrm{vWF}$ and P-selectin in LAA tissue with RT-PCR has not been previously reported. The expression of the vWF gene in the LAA was significantly increased in patients with MS combined with AF, and this was more obvious in patients who also had LAT. The changes in vWF gene expression in the LAA samples from different groups were related to plasma vWF concentrations. The overexpression of the vWF gene in the LAA could be an important contributor to the elevation of plasma $\mathrm{vWF}$ concentration.

Literature review did not find any reports on P-selectin gene expression in LAA tissue. In an immunohistochemical study, we found P-selectin protein to be immunostained

TABLE 3. Normalized von Willebrand factor gene expression in left atrial appendage specimens

\begin{tabular}{lcccccc}
\hline & & & & & \multicolumn{2}{c}{ Normalized vWF gene } \\
& Cases & $\mathbf{C t}_{\mathbf{v W F} \text { gene }}$ & $\mathbf{C t}_{\text {GAPDH gene }}$ & $\boldsymbol{\Delta C t}$ & $\boldsymbol{\Delta \Delta C t}$ & 3.04 \\
\hline Patients with LAT & 40 & $17.54 \pm 1.52$ & $16.92 \pm 1.51$ & $0.63 \pm 0.26$ & -1.60 & 2.16 \\
Patients without LAT & 40 & $17.51 \pm 1.87$ & $16.39 \pm 1.95$ & $1.12 \pm 0.46$ & -1.11 & 1.00 \\
Control & 10 & $17.67 \pm 1.36$ & $15.44 \pm 1.25$ & $2.23 \pm 0.45$ & 0 & \\
\hline
\end{tabular}

Difference between groups was statistically significant $(P<.001)$. Values for all but $\triangle \Delta \mathrm{Ct}$ are mean $\pm \mathrm{SD}$. Ct, Threshold cycle; $v W F$, von Willebrand factor; GAPDH, glyceraldehyde 3-phosphate dehydrogenase; $\Delta C t$, derived target gene value $\left(\mathrm{Ct}_{\text {Target gene }}-\mathrm{Ct}_{\mathrm{H}_{\text {Housekeeping gene }}}\right) ; \Delta \Delta C t$, derived group difference value $\left(\Delta \mathrm{Ct}\right.$ Study group $\left.-\Delta \Delta \mathrm{Ct}_{\mathrm{Control} \mathrm{group}}\right)$; $L A T$, left atrial thrombosis. 
TABLE 4. Normalized P-selectin gene expression in left atrial appendage specimens

\begin{tabular}{lcccccc}
\hline & Cases & $\mathbf{C t}_{\text {P-selectin gene }}$ & $\mathbf{C t}_{\text {GAPDH gene }}$ & $\boldsymbol{\Delta C t}$ & $\boldsymbol{\Delta \Delta C t}$ & $\begin{array}{c}\text { Normalized P-selectin gene } \\
\text { expression }\end{array}$ \\
\hline Patients with LAT & 40 & $27.98 \pm 1.94$ & $16.92 \pm 1.51$ & $11.06 \pm 1.33$ & -0.22 & 1.16 \\
Patients without LAT & 40 & $27.25 \pm 2.04$ & $16.39 \pm 1.95$ & $10.86 \pm 1.42$ & -0.42 & 1.34 \\
Control & 10 & $26.72 \pm 2.06$ & $15.44 \pm 1.25$ & $11.28 \pm 1.84$ & 0 & 1.00 \\
\hline
\end{tabular}

Difference between groups was not statistically significant $(P=.9850)$. $C t$, Threshold cycle; $G A P D H$, glyceraldehyde 3-phosphate dehydrogenase; $\triangle C t$, derived target gene value $\left(\mathrm{Ct}_{\text {Target gene }}-\mathrm{Ct}_{\text {Housekeeping gene }}\right) ; \Delta \Delta C t$, derived group difference value $\left(\Delta \mathrm{Ct}_{\text {Study group }}-\Delta \Delta \mathrm{Ct}_{\mathrm{Control} \mathrm{group}}\right) ; L A T$, left atrial thrombosis.

both in the left atrial endocardium and inside the cardiomyocytes. RT-PCR was therefore carried out to investigate the expression of P-selectin in LAA tissue. Although plasma P-selectin concentration was increased in patients with MS combined with AF, there was no significant difference in P-selectin expression in LAA between the groups. The changes of plasma P-selectin levels had no relation to changes in P-selectin gene expression in the LAA or blood platelet count. Other pathways involved in abnormal platelet activation that cause increased plasma $\mathrm{P}$-selectin concentration should be further investigated.

Previous studies confirmed that the changes in plasma vWF level are linked with P-selectin levels. They have been considered as independent predictors of cardiovascular events and stroke in patients with $\mathrm{AF}^{23,26}$ Our results demonstrate a similar trend in the changes of plasma vWF and P-selectin levels in patients with MS combined with AF. Plasma levels of vWF and P-selectin in patients with MS who also had LAT were found to be significantly higher than in those patients without LAT and in healthy subjects. Conversely, plasma levels of vWF and P-selectin in patients with MS without LAT were shown to be higher than those in healthy subjects. Patients with LAT had a larger left atrial dimension, smaller mitral valve orifice area, and longer duration of $\mathrm{AF}$, which means their disease was more serious than that in patients without LAT. The increased vWF, in association with the disruption of the endocardium, would contribute to the initiation of thrombogenesis by promoting platelet adhesion and aggregation on the endocardial surface to form a platelet membrane. The elevated P-selectin would guide more platelet aggregation, thus causing the thrombus to grow further into the atrial lumen. When significant endothelial dysfunction and platelet activation occur in patients with MS and AF, vWF and P-selectin may play a collaborative role in promoting thrombosis as a response to endothelial injury in the LAA. Monitoring of plasma vWF and P-selectin levels would be helpful in delineating the extent of endocardial dysfunction and platelet activation. Perhaps stronger antiplatelet therapy, such as dual antiplatelet therapy with clopidogrel and aspirin, would be beneficial for patients with MS and AF. Further clinical studies are required.

Some limitations exist in this clinical trial. First, it represents a small research cohort. The definite time of
LAT formation is unclear, and the size of LAT is difficult to measure during surgery because of its fragility. It is very difficult to subcategorize the LAT group with regard to degree of clot burden. Second, this is an initial result of our research. We found that $\mathrm{vWF}$ and P-selectin may play a collaborative role in thrombosis related to endothelial injury. Any strategy of stronger antiplatelet therapy for these patients, however, needs to be confirmed with further clinical studies. Another research study has reported that the combination of antiplatelet therapy plus moderate intensity anticoagulation for patients with AF safely and significantly decreases the vascular events seen with anticoagulation alone. $^{27}$

\section{CONCLUSIONS}

Left atrial endocardial dysfunction and platelet activation are present in patients with MS combined with AF. No differences were observed in plasma vWF and P-selectin concentrations between left atrial and peripheral venous blood. The overexpression of the vWF gene in the LAA may contribute to the increase of plasma vWF levels. Possibly vWF and P-selectin play a collaborative role to thrombosis in response to endothelial injury in LAA.

\section{References}

1. Shrestha NK, Moreno FL, Narciso FV, Torres L, Calleja HB. Two-dimensional echocardiographic diagnosis of left-atrial thrombus in rheumatic heart disease. A clinicopathologic study. Circulation. 1983;67:341-7.

2. Hao XH, Lai YQ, Li JH, Dai J, Song BR, Zhang ZG. Analysis of risk factors for left atrial thrombosis in patients with rheumatic mitral stenosis [in Chinese] Chinese J Thoracic Cardiovascular Surg. 2011;27:293-6.

3. Goldsmith I, Kumar P, Carter P, Blann AD, Patel RL, Lip GY. Atrial endocardial changes in mitral valve disease: a scanning electron microscopy study. Am Heart J. 2000;140:777-84.

4. Shirani J, Alaeddini J. Structural remodeling of the left atrial appendage in patients with chronic non-valvular atrial fibrillation: implications for thrombus formation, systemic embolism, and assessment by transesophageal echocardiography. Cardiovasc Pathol. 2000;9:95-101.

5. Marin F, Roldán V, Lip GY. Fibrinolytic function and atrial fibrillation. Thromb Res. 2003;109:233-40.

6. Freestone B, Krishnamoorthy S, Lip GY. Assessment of endothelial dysfunction. Expert Rev Cardiovasc Ther. 2010;8:557-71.

7. Doty JR, Doty DB, Jones KW, Flores JH, Mensah M, Reid BB, et al. Comparison of standard Maze III and radiofrequency Maze operation for treatment of atrial fibrillation. J Thorac Cardiovasc Surg. 2007;133:1037-44.

8. Anné W, Willems R, Roskams T, Sergeant P, Herijgers P, Holemans P, et al. Matrix metalloproteinases and atrial remodeling in patients with mitral valve disease and atrial fibrillation. Cardiovasc Res. 2005;67:655-66.

9. Watson T, Shantsila E, Lip GY. Mechanisms of thrombogenesis in atrial fibrillation: Virchow's triad revisited. Lancet. 2009;373:155-66. 
10. Conway DS, Pearce LA, Chin BS, Hart RG, Lip GY. Plasma von Willebrand factor and soluble P-selectin as indices of endothelial damage and platelet activation in 1321 patients with nonvalvular atrial fibrillation: relationship to stroke risk factors. Circulation. 2002;106:1962-7.

11. Kim R, Baumgartner N, Clements J. Routine left atrial appendage ligation during cardiac surgery may prevent postoperative atrial fibrillation-related cerebrovascular accident. J Thorac Cardiovasc Surg. 2013;145:582-9; discussion 589.

12. Choi BW, Bacharach SL, Barbour DJ, Leon MB, McCarthy KE, Bonow RO. Left ventricular systolic dysfunction diastolic filling characteristics and exercise cardiac reserve in mitral stenosis. Am J Cardiol. 1995;75:526-9.

13. Kamp O, Verhorst PM, Welling RC, Visser CA. Importance of left atrial appendage flow as a predictor of thromboembolic events in patients with atrial fibrillation. Eur Heart J. 1999;20:979-85.

14. Laroia ST, Ganti AK, Laroia AT, Tendulkar KK. Endothelium and the lipid metabolism: the current understanding. Int J Cardiol. 2003;88:1-9.

15. Leroyer AS, Anfosso F, Lacroix R, Sabatier F, Simoncini S, Njock SM, et al. Endothelial-derived microparticles: biological conveyors at the crossroad of inflammation, thrombosis and angiogenesis. Thromb Haemost. 2010;104: 456-63.

16. Geuzebroek GS, van Amersfoorth SC, Hoogendijk MG, Kelder JC, van Hemel NM, de Bakker JM, et al. Increased amount of atrial fibrosis in patients with atrial fibrillation secondary to mitral valve disease. J Thorac Cardiovasc Surg. 2012;144:327-33.

17. Schumacher A, Seljeflot I, Sommervoll L, Christensen B, Otterstad JE, Arnesen H. Increased levels of endothelial haemostatic markers in patients with coronary heart disease. Thromb Res. 2002;105:25-31.
18. Krishnamoorthy S, Lim SH, Lip GY. Assessment of endothelial (dys)function in atrial fibrillation. Ann Med. 2009;41:576-90.

19. Merten M, Thiagarajan P. P-selectin expression on platelets determines size and stability of platelet aggregates. Circulation. 2000;102:1931-6.

20. Tamagawa-Mineoka R, Katoh N, Kishimoto S. Platelet activation in patients with psoriasis: increased plasma levels of platelet-derived microparticles and soluble P-selectin. J Am Acad Dermatol. 2010;62:621-6.

21. Gasparyan AY. Platelets in inflammation and thrombosis. Inflamm Allergy Drug Targets. 2010;9:319-21.

22. Chen MC, Wu CJ, Yip HK, Chang HW, Fang CY, Yu TH, et al. Left atrial platelet activity with rheumatic mitral stenosis: correlation study of severity and platelet P-selectin expression by flow cytometry. Chest. 2003;124:1663-9.

23. Chen MC, Chang HW, Juang SS, Yip HK, Wu CJ. Increased plasma levels of soluble P-selectin in rheumatic mitral stenosis. Chest. 2004;126:54-8.

24. Kumagai K, Fukuchi M, Ohta J, Baba S, Oda K, Akimoto H, et al. Expression of the von Willebrand factor in atrial endocardium is increased in atrial fibrillation depending on the extent of structural remodeling. Circ J. 2004;68:321-7.

25. Freestone B, Chong AY, Nuttall S, Blann AD, Lip GY. Soluble E-selectin, von Willebrand factor, soluble thrombomodulin, and total body nitrate/nitrite product as indices of endothelial damage/dysfunction in paroxysmal, persistent, and permanent atrial fibrillation. Chest. 2007;132:1253-8.

26. Conway DS, Pearce LA, Chin BS, Hart RG, Lip GY. Prognostic value of plasma von Willebrand factor and soluble P-selectin as indices of endothelial damage and platelet activation in 994 patients with nonvalvular atrial fibrillation. Circulation. 2003;107:3141-5.

27. Lip GY, Boos CJ. Antithrombotic treatment in atrial fibrillation. Heart. 2006;92: 155-61.

\title{
EDITORIAL COMMENTARY
}

\section{Atrial thrombosis in advanced mitral stenosis with atrial fibrillation: What should we expect?}

\author{
Carlos-A. Mestres, MD, PhD, FETCS
}

See related article on pages 1970-6.

In this issue of the Journal of Thoracic and Cardiovascular Surgery, Luo and colleagues ${ }^{1}$ publish their study intended to evaluate the role of left atrial endocardial dysfunction and platelet activation in left atrial thrombosis (LAT). Their study included 80 patients with rheumatic mitral stenosis

From the Cardiovascular and Thoracic Surgery, Heart and Vascular Institute, Cleveland Clinic Abu Dhabi, Abu Dhabi, United Arab Emirates.

Disclosures: Dr Mestres reports consulting fees from Edwards Lifesciences, lecture fees from On-X Life Technologies, and equity ownership in Symetis SA.

Received for publication Aug 21, 2014; accepted for publication Aug 23, 2014; available ahead of print Sept 26, 2014.

Address for reprints: Carlos-A. Mestres, MD, PhD, FETCS, Heart and Vascular Institute, Cleveland Clinic Abu Dhabi, Sowwah Square, Al Maryah Island, PO Box 112412, Abu Dhabi, United Arab Emirates (E-mail: MestreC@ ClevelandClinicAbuDhabi.ae).

J Thorac Cardiovasc Surg 2014;148:1976-7

$0022-5223 / \$ 36.00$

Copyright (c) 2014 by The American Association for Thoracic Surgery

http://dx.doi.org/10.1016/j.jtcvs.2014.08.041

(MS) and atrial fibrillation (AF) with (40 patients) or without (40 patients) LAT. They also evaluated the blood of 15 apparently healthy volunteers and 10 specimens of left atrial appendages harvested from donor hearts, looking at the expressions as well as plasma concentrations of von Willebrand factor (vWF) and P-selectin protein. Patients with LAT had bigger left atrial dimensions and smaller valve orifices. Luo and colleagues ${ }^{1}$ found no differences in plasma levels of vWF and P-selectin between the left atrium and peripheral blood. They concluded that the overexpression of the $\mathrm{vWF}$ gene in the left atrial appendage may contribute to the increase in plasma vWF levels. What is extracted from this contribution is that $\mathrm{VWF}$ and $\mathrm{P}$-selectin are supposed to play a collaborative role in thrombosis on the injured endothelium in left atrial appendage.

Rheumatic MS as a sequela of acute rheumatic fever is a still common disease in various parts of the world, ${ }^{2}$ and because it is a chronic inflammatory disease, a number of complex phenomena are observed. Subclinical chronic carditis has been previously confirmed by Chopra and 